\title{
Investigation of Compound Relaxation Processes in Crystal Lattice Dynamics of Si Irradiated by Soft X-rays
}

\author{
A.J. JanaviČIUS ${ }^{a}$, R. PURlys ${ }^{b}$ AND Ž. NorgÉla ${ }^{a}$ \\ ${ }^{a}$ Šiauliai University, P. Višinskio 25, 76351 Šiauliai, Lithuania \\ ${ }^{b}$ Faculty of Physics, Vilnius University, Saulètekio 9, 10222 Vilnius, Lithuania
}

(Received April 13, 2005; revised version September 20, 2005)

\begin{abstract}
We applied soft X-rays for investigation of dynamics of Frenkel point defects in a Si crystal during its saturation with metastable vacancies with neighboring $\mathrm{Si}$ atoms in excited states or vacancies with neighboring Si atoms in interstitial states produced in the lattice after ejection of Auger electrons. The irradiated irregularities and defects of the lattice cause a change of Bragg reflection maxima. Several resonance phenomena related to the metastable states introduced into Si crystal by soft X-rays irradiation have been detected.
\end{abstract}

PACS numbers: 61.72.- - , 61.72.Dd, 05.50.+q

\section{Introduction}

The existence of relaxation processes of Frenkel point defects and very fast diffusion of metastable lattice vacancies [1] in a Si crystal irradiated by soft X-rays were found and presented in papers [2,3]. This very important phenomenon was detected by investigation of the time dependence of Hall mobility in a crystalline $\mathrm{Si}$ sample after switching off soft X-ray irradiation. The complicated time dependence of current and Hall mobility and abrupt changes of these properties after $1500 \mathrm{~min}$ since the moment of switch-off indicate that relaxation processes associated with metastable defects in the silicon are involved.

The aim of this investigation is to detect these relaxation processes of metastable states, using measurements of the time dependence of the diffraction maximum intensity of reflection spectrum index (333) from a crystal of orientation (111) and size $0.0170 \times 0.004 \times 0.004 \mathrm{~m}^{3}$. Measurements were done with a diffractometer DRON-2 (Russian device). The intensity of irradiation was registered by a LiF dosimeter and doses of irradiation were measured by the thermoluminescent dosimeter (TLD) device [4] manufactured in the factory "Rodos" in Finland. 
It is known that Frenkel point defects and vibration of the crystal lattice influence the intensity of interference lines of the diffracted X-rays and diffusion background. By measuring relative intensity of X-ray reflection at a fixed diffraction angle $\vartheta=47.48^{\circ}$ for the $K_{\alpha 1}$ line of $\mathrm{Cu}$ X-ray characteristic spectrum (with the wavelength $\lambda=1.5405 \AA$ ) at two different energies of irradiation, we obtained the mean square displacements $\left\langle u_{\mathrm{s}}^{2}\right\rangle$ of $\mathrm{Si}$ atoms from their regular equilibrium positions in a crystal lattice. The resonance-like behavior of the time dependence of $\left\langle u_{\mathrm{s}}^{2}\right\rangle$ can be explained by the presence of excited atoms in the metastable states [1] in a Si crystal irradiated by soft X-rays.

\section{Measurements of dynamics of Frenkel point defects in Si crystal irradiated by soft X-rays}

Quantitative analysis of crystalline structure is based on the investigations of intensity [5] $I_{h k l}$ of X-rays reflected from the crystal lattice, with reflection indexes $h k l$ :

$$
I_{h k l}=I_{0} c F_{h k l}^{2} L P A(\vartheta, \mu) p V,
$$

where $I_{0}$ is the intensity of incident beam, $c$ is a constant, $F_{h k l}^{2}$ is the structure factor, $L P$ is the product of Lorenz and polarization factors, $A$ is the absorption factor, $p$ is the multiplicity factor, $V$ is the scattering volume. Thermal motion and displacement of atoms to metastable or interstitial positions in the crystal lattice caused by Auger effect [5] are changing the structure factor [5]:

$$
F_{h k l}^{2}=F_{0}^{2} \exp \left(-\frac{16}{3} \pi^{2}\left\langle u^{2}\right\rangle(\sin \vartheta / \lambda)^{2}\right) .
$$

Here, $F_{0}$ is the structure factor for $0 \mathrm{~K}$ temperature, in the absence of structural defects, $\sqrt{\left\langle u^{2}\right\rangle}$ is a square root of mean square deviation of atoms from regular pattern.

Since the changes in a crystal lattice generated by thermal motion and irradiation of defects are independent processes, we have

$$
\left\langle u^{2}\right\rangle=\left\langle u_{\mathrm{t}}^{2}\right\rangle+\left\langle u_{\mathrm{d}}^{2}\right\rangle,
$$

where $\sqrt{\left\langle u_{\mathrm{t}}^{2}\right\rangle}$ and $\sqrt{\left\langle u_{\mathrm{d}}^{2}\right\rangle}$ are root-mean-square deviations of atoms from regular positions, caused by thermal motion and X-ray-generated defects, respectively. Since all measurements were done at room temperature, changes in relative intensity $I / I_{0}$ of diffraction maximum can be related to changes of $\sqrt{\left\langle u_{\mathrm{d}}^{2}\right\rangle}$ or to amount of point defects generated by the soft X-rays:

$$
\frac{I}{I_{0}}=\exp \left(-\frac{16}{3} \pi^{2}\left\langle u_{\mathrm{d}}^{2}\right\rangle(\sin \vartheta / \lambda)^{2}\right),
$$

from which we obtain

$$
\sqrt{\left\langle u_{\mathrm{d}}^{2}\right\rangle}=\frac{\sqrt{\ln \frac{I_{0}}{I}}}{\frac{4 \pi}{\sqrt{3}} \frac{\sin \vartheta}{\lambda}} .
$$


In the case of the Auger effect, kinetic energy of the ejected electron, called an Auger electron, is equal to the energy of the corresponding X-ray photon minus the electron binding energy. The highest probability of the Auger effect and the largest amount of point defects produced by this effect in Si crystal can be received in the case of X-ray photons with $6 \mathrm{keV}$ energy [6]. The Frenkel point defects are obtained as a result of Coulomb-force interactions and transitions of the ionized $\mathrm{Si}$ atoms from their equilibrium sites to metastable or interstitial states. The relative fluctuations of the number of atoms in excited states can be related to relative fluctuations of crystal volume containing $N_{\mathrm{mi}}$ atoms:

$$
\Delta V(t) \approx N_{\mathrm{mi}}(t) a^{3} \text {. }
$$

Here, $a=5.431 \AA$ is the Si lattice constant in vacuum at $295.6 \mathrm{~K}$.

Taking into account the fact that fluctuations of the crystal volume can be related to changes of $\sqrt{\left\langle u_{\mathrm{d}}^{2}\right\rangle}$, we obtain

$$
\Delta V(t) \approx N\left(\sqrt{\left\langle u_{\mathrm{d}}^{2}\right\rangle(t)}\right)^{3},
$$

where $N=5 \times 10^{22} \mathrm{~cm}^{-3}$ is the number of Si atoms in the unit volume. From the formulae (2.6) and (2.7) we obtain

$$
N_{\mathrm{mi}}(t) \approx N\left(\sqrt{\left\langle u_{\mathrm{d}}^{2}\right\rangle(t)}\right)^{3} / a^{3} .
$$

For $\mathrm{Cu}$ anode, the maximum spectral density of continuous X-ray spectrum at photon energy $6 \mathrm{keV}$ is achieved when the X-ray tube voltage is equal to $9 \mathrm{kV}$.

After long irradiation of 6-12 hours of the sample, we detected a decrease in maximum intensity without changing half of width of diffraction peak. This indicates that the X-rays generate only point defects [5]. After irradiation of 50-70 hours, the crystal lattice of $\mathrm{Si}$ is restored. This fact shows that metastable vacancies and excited point defects in Si crystals can be generated not only by the action of $1-2 \mathrm{MeV}$ electrons [7] or ${ }^{60} \mathrm{Co}$ gamma-rays [8] but also with soft X-rays $[2,3]$. The recovery of Si crystal after irradiation is not complete and this fact is related to interstitial atoms or some stable complexes, whose amount increases during irradiation. Consequently, we can express the mean square deviation of lattice from the regular pattern, resulting from atom transitions into metastable or interstitial states, in the following way:

$$
\left\langle u_{\mathrm{d}}^{2}\right\rangle=\left\langle u_{\mathrm{dv}}^{2}\right\rangle+\left\langle u_{\mathrm{di}}^{2}\right\rangle .
$$

The magnitude of $\left\langle u_{\mathrm{dv}}^{2}\right\rangle$ depends on the number of excited vacancies and atoms in metastable states, and $\left\langle u_{\mathrm{di}}^{2}\right\rangle$ depends on the number of interstitial atoms. The number of interstitial atoms is proportional to irradiation dose.

The vacancy formation energy can be calculated as follows [9]:

$$
\Delta E=E_{\mathrm{v}}-\frac{n-1}{n} E_{\mathrm{b}},
$$

where $E_{\mathrm{v}}$ is the total energy of the cell containing the vacancy, $n$ is the number of atoms in the cell, and $E_{\mathrm{b}}$ denotes the total energy of the bulk calculated us- 
ing the same cell parameters. When after the irradiation the crystal lattice has a non-equilibrium number of interstitial atoms and associated vacancies, the crystal is in the excited state and energy of the crystal is increased by $n_{\mathrm{vi}} \Delta E_{\mathrm{i}}$. Here $n_{\mathrm{vi}}$ and $\Delta E_{\mathrm{i}}$ are the number and the energy of the produced pairs of interstitial atoms and excited vacancies in the process of irradiation. As a result of energy release in the carriers capture process by vacancies and vacancies recombination with the interstitial atoms, we have energy convertion to the vibrational modes of Si lattice. Also the crystal energy increases on $n_{\mathrm{ve}} \Delta E_{\mathrm{e}}$ by the Auger effect as a result of generation of $n_{\mathrm{ve}}$ excited vacancies with neighboring atom in the excited state. The total energy $\Delta E_{\mathrm{f}}$ absorbed in the crystal can be approximately expressed as the sum of the two terms involving generation energies of Frenkel point defects and the term reflecting energy loss in the process of crystal lattice transition into the thermal equilibrium state:

$$
\Delta E_{\mathrm{f}}(t)=n(t)_{\mathrm{ei}} \Delta E_{\mathrm{i}}+n(t)_{\mathrm{ve}} \Delta E_{\mathrm{e}}+\Delta E_{\mathrm{m}}(t) .
$$

The term $\Delta E_{\mathrm{m}}(t)$ represents the time dependent energy loss related to transitions of metastable states of Frenkel point defects into stable states. The numbers of atoms in metastable and interstitial states depend on time in a different manner, and we can measure a relaxation process of the relative intensity $I / I_{0}$ of diffraction maximum caused by time dependence of the number of Frenkel point defects composed of the vacancies and neighboring atoms in metastable or interstitial states.

\section{Compound relaxation processes in excited systems}

For investigation of the temperature dependence of kinetic parameters (time, frequency, concentration, parameters of diffusion, and Brownian motion) of compound systems, the Arrhenius law is usually applied [4]:

$$
\Phi_{n}(T)=A \exp \left( \pm U_{n} / k T\right) .
$$

The $U_{n}$ terms can be determined from the experimental measurements of $\ln \Phi_{n}(1 / T)$. The usually obtained values of $U_{n}$ are $5-10 \mathrm{eV}$. These values are larger than the true interaction energy between atoms or molecules in condensed systems, where this term is usually equal to about $1 \mathrm{eV}$. This can be explained only when $U_{n}$ is not a potential barrier for elementary event, but a sum of potential barriers of elementary acts of compound collective processes instead [4].

In this case, the probability of producing vacancies by compound processes caused by irradiation of Si crystal with soft X-rays can be represented by the following formula [4]:

$$
W=\sum_{n} W_{n} \exp \left(-E_{n} / k T\right) .
$$

Here, $W_{n}$ is a probability of transition from the crystal site with energy $E_{n}$ to the interstitial or metastable state [1]. This formula can also describe the reverse process. In the case of a two-level system, we can use the Boltzmann statistics for 
calculation of population ratio [10] $P$ of two quantum levels with energies $\varepsilon_{2}>\varepsilon_{1}$

$$
P=\frac{\omega\left(\varepsilon_{2}\right)}{\omega\left(\varepsilon_{1}\right)}=\exp \left(\frac{-E_{21}}{k T}\right),
$$

where $E_{21}=\varepsilon_{2}-\varepsilon_{1}, E_{21}>0$. For an excited system, $P \geq 1$, and then we have $T<0$.

It is possible to describe properties of an excited system in the non-isothermal case by introducing the following temperature function [10]:

$$
T(t)=\frac{1}{a-b \ln (t c)} .
$$

The temperature function $T(t)$ depends on parameters $a, b, c$ and has a singularity at the time moment $t_{0}$. We can express time $t$ in the units of time moment $t_{0}$, then we obtain

$$
t=z t_{0}, \quad T(t)=-\frac{1}{b \ln (z)}, \quad t_{0}=\frac{1}{c} \exp (a / b) .
$$

It is very important that the temperature function $T(t)$ corresponds to the Boltzmann statistics for description of the population ratio $(3.3) P(t)$ of two quantum levels $E_{2}>E_{1}$ :

$$
P(t)=z^{E_{21} b / k}=z^{\alpha-1}, \quad \alpha=1+\frac{E b}{k} .
$$

$E$ is the activation energy of the diffusion in solids.

From the last expression, if $b<0$ and $\alpha<1$, we obtain that all excited vibration states $E_{2}$ of atoms taking part in diffusion are populated at the initial time when $z \rightarrow 0$. When $b>0$ and $\alpha>1$, we have the process of saturation of the system by the excited states. The parameter $b$ depends on intensity and spectrum of X-rays used for irradiation of the crystal. Vacancies are produced by moving the atoms from the lattice site to metastable state [4] with $\epsilon_{2}=1 \mathrm{eV}$ energy or to the interstitial state as a result of Coulomb interaction after the ejection of Auger electrons. For displacement of these atoms to metastable states, they must penetrate through the barrier of height $\Delta E=1.5 \mathrm{eV}[5]$, and for displacement to interstitial state [2] with an energy of $0.8 \mathrm{eV}$, they must penetrate through the additional potential barrier of height [5] $0.5 \mathrm{eV}$.

Then, using (3.3) and (3.6), we obtain

$$
\frac{N_{m}(z)}{N-N_{m}(z)}=z^{\alpha-1} \text {. }
$$

Here, $N_{m}$ is the number of Si atoms in metastable and interstitial states, $N$ is the number of $\mathrm{Si}$ atoms in $\mathrm{cm}^{3}$. The last formula can be easily transformed to a more handy form:

$$
\frac{N_{m}(z)}{N}=\frac{z^{\alpha-1}}{z^{\alpha-1}+1} .
$$

If we have periodic excitation of metastable system with a relaxation period $\tau_{\mathrm{i}}=t_{\mathrm{f}}-t_{\mathrm{i}}$, we can present (3.8) in the following way: 


$$
\frac{N_{m}(t)}{N}=\frac{\left(\frac{t-t_{\mathrm{f}}}{t_{0}}\right)^{\alpha-1}}{\left(\frac{t-t_{\mathrm{i}}}{t_{0}}\right)^{\alpha 1}+1}, \quad t_{\mathrm{i}} \leq t \leq t_{\mathrm{f}}
$$

\section{Relaxation processes in crystalline silicon initiated by soft X-rays}

In our experiments we used the crystal sample of high-resistance $667 \Omega \mathrm{cm}$ $\mathrm{Si}$ doped with boron. If the Si crystal is doped with boron the three electrons form a covalent bond with neighboring $\mathrm{Si}$ atoms, leaving the fourth bond broken. The irradiation of the Si crystal with soft X-rays initiates Auger processes which generates the very fast vacancies $[2,3]$ and breaks bonds in the lattice. All broken bonds change the energy of lattice and increase lattice distortions. Carrier density dependence on Auger recombination, carriers transitions to charged vacancies and phonon-assisted Auger recombination [11] make expedient to investigate the influence of these processes dependence of lattice parameters on irradiation. Fast non-radiative Auger recombination and free carriers absorption prevent optical transitions in crystal silicon [12] and increase probability of relaxation effects in lattice. These relaxation effects also can be generated by the different diffusivity of vacancies in different charge states. For diffusion of charged vacancies like in diffusion experiments [13] with the Si samples doped by $\mathrm{B}$ and $\mathrm{P}$ the Fermi level $E_{\mathrm{F}}$ must depend on time. It is important that concentration of the charged defects also vary [13] with $E_{\mathrm{F}}$.

Experimental measurements of intensity of X-ray reflection were performed with a diffractometer DRON-2 by summing the number of photons in $10 \mathrm{~s}$ time interval with $1 \mathrm{~min}$ period for a fixed diffraction angle $\vartheta=47.48^{\circ}$. The experiment was done using a $\mathrm{Cu}$ anode with X-ray characteristic spectral line $K_{\alpha 1}$ of wavelength $\lambda=1.5405 \AA$ for anode voltages $10.4 \mathrm{kV}, 13 \mathrm{kV}$ and Roentgen tube currents $30 \mathrm{~mA}, 16 \mathrm{~mA}$, respectively. Results are presented in Fig. 1 and Fig. 3. We calculated and presented in Fig. 2 and Fig. 4 the square root $i=\sqrt{\left\langle u_{\mathrm{d}}\right\rangle^{2}}$ of the mean square displacements (2.5) of Si atoms from their regular equilibrium positions in crystal lattice dependence on time of irradiation. The oscillating terms $i$ of these displacements were fitted with the formula (3.9) for the time dependence of saturation of Si crystal lattice with metastable states, using a fixed value of the excitation parameter $\alpha=0.8$. These oscillations with the same excitation parameter $\alpha$ show that Si crystal lattice irradiated by soft X-rays acts as a two-level system [10] for atomic transitions from metastable states into neighboring metastable vacancies or the reverse transitions by producing metastable vacancies. Also can be produced the simple vacancies with neighboring atoms in the interstitial sites. All these processes are generated by electron transitions between conductivity band and charged vacancies and excited atoms vibrations in metastable states. The importance of excited lattice vibrations can be derived from the fact that Si crystal after irradiation can stay in excited states many hours $[2,3]$. The initial part of dependence of the point defect-producing process 


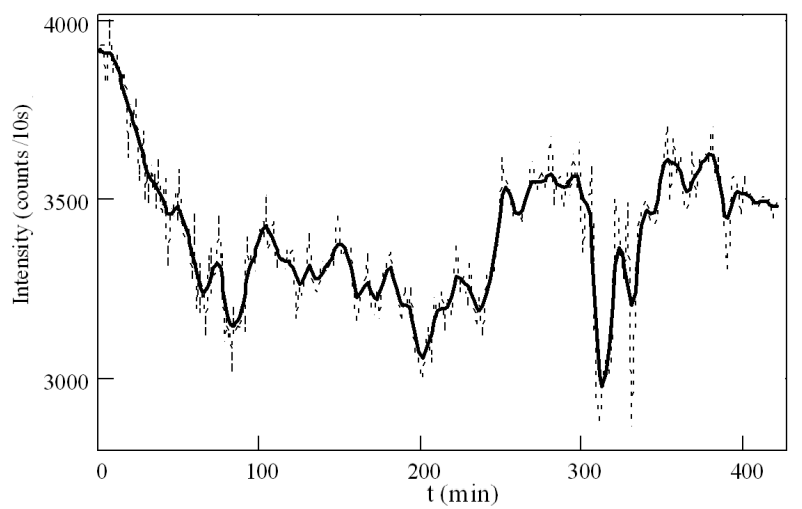

Fig. 1. Experimental dependence of intensity (number of photons/10 seconds) of reflection from Si crystal orientated (111) on time (with diffractometer DRON-2 for a fixed diffraction angle $\vartheta=47.48^{\circ}$ ). Experiment was done with $\mathrm{Cu}$ anode $\mathrm{X}$-ray characteristic spectral line $K_{\alpha 1}$ of wavelength $\lambda=1.5405 \AA$ for anode voltage $10.4 \mathrm{kV}$ and X-ray tube current $30 \mathrm{~mA}$.

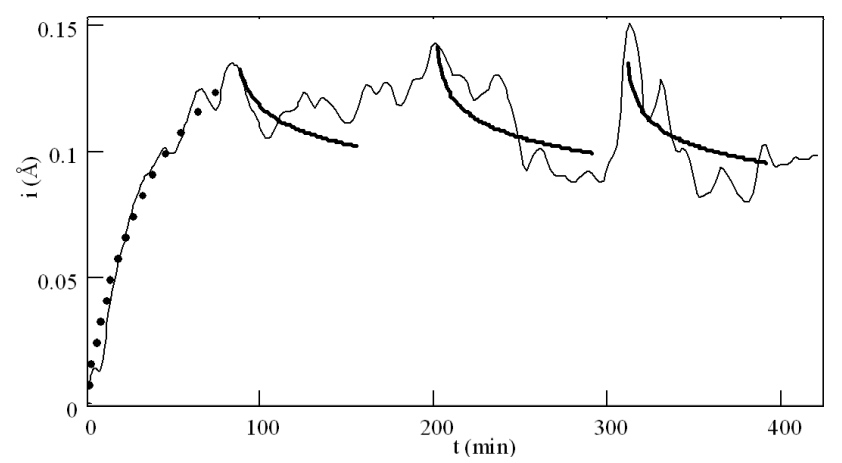

Fig. 2. Dependence of square root of mean square displacements $i=\sqrt{\left\langle u_{\mathrm{d}}^{2}\right\rangle}$ of atoms from equilibrium positions on time $t$. Bold lines are interpolations of $i$ and describe periodic transitions $(\alpha=0.8)$ from metastable states (3.9) with equal time periods $t_{0}=41 \mathrm{~min}$ into lower metastable state of Si crystal. The interpolation line (dots) is proportional to the excitation function (3.9) with excitation parameter $\alpha=1.77$ and has a period of $t_{0}=90 \mathrm{~min}$. X-ray tube current was $30 \mathrm{~mA}$, anode voltage was $10.4 \mathrm{kV}$.

on irradiation time for both experiments can be defined by the same excitation parameter $\alpha=1.77$. The equal constants of excitation for different anode voltages and Roentgen tube currents demonstrate that these constants depend only on properties of Si crystal. The obtained resonance of the term $i$ can be interpreted as normal modes of resonant transitions of coupled oscillating atoms into metastables states and the reverse transitions into vacancies in the process of the irradiation by 5-15 keV photons. These photons can increase the vibration energy of the lattice and produce excited vacancies by means of Auger effect. The vibrations of atoms 


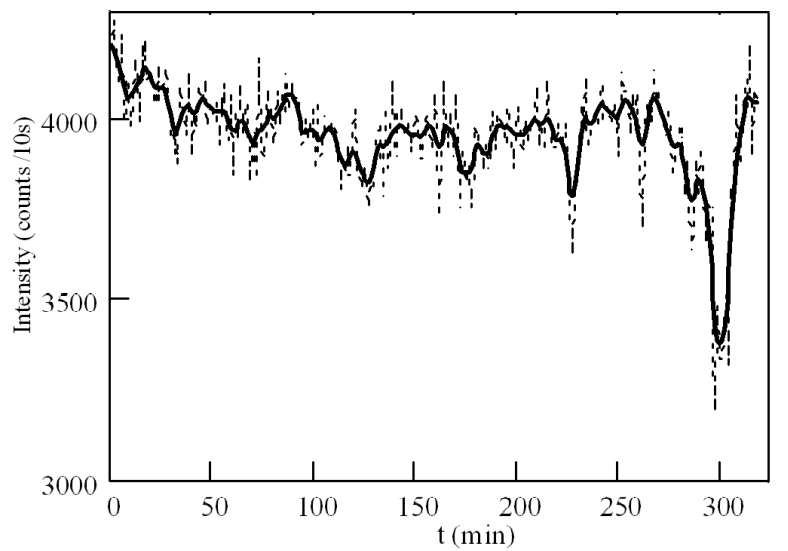

Fig. 3. Experimental dependence of intensity (number of photons/10 seconds) of reflection from Si crystal orientated (111) on time (with diffractometer DRON-2 for a fixed diffraction angle $\vartheta=47.48^{\circ}$ ). Experiment was done with $\mathrm{Cu}$ anode X-ray characteristic spectral line $K_{\alpha 1}$ of wavelength $\lambda=1.5405 \AA$ for anode voltage $13 \mathrm{kV}$ and X-ray tube current $16 \mathrm{~mA}$.

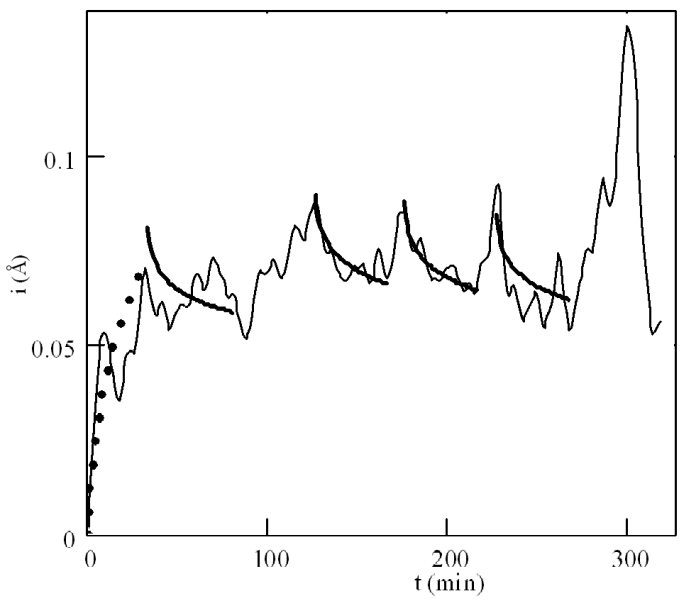

Fig. 4. Dependence of square root of mean square displacements $i=\sqrt{\left\langle u_{\mathrm{d}}^{2}\right\rangle}$ of atoms from equilibrium positions on time $t$. Bold lines are interpolations of $i$ and describe periodic transitions $(\alpha=0.8)$ from metastable states (3.9) with equal time periods $t_{0}=41 \mathrm{~min}$ into lower metastable states of Si crystal. The interpolation line (dots) is proportional to the excitation function (3.9) with excitation parameter $\alpha=1.77$ and has a period of $t_{0}=33 \mathrm{~min}$. X-ray tube current was $16 \mathrm{~mA}$, anode voltage was $13 \mathrm{kV}$.

in excited states of lattice generated by X-rays play the role of external periodic force, and when its frequency coincides with the frequency of thermal vibrations of atoms placed at neighboring of excited vacancies [14] $\omega_{\mathrm{v}}=\sqrt{3 k / m^{-\gamma^{2}}}$, the res- 
onance occurs. Here, $k$ is oscillators constant, $\gamma=c / 2 m$, where $c$ is the coefficient of velocity-dependent damping force, $m$ is the oscillators mass. The maximum amplitude of oscillations of vacancies of neighboring atoms occurs at the resonant frequency $\omega_{\mathrm{r}}=\omega_{\mathrm{v}}$. This resonance can be obtained only for special values of anode current. The probability of Auger effect and vacancy formation depends on the anode voltage. It follows that oscillations of the number of point defects in $\mathrm{Si}$ latice can be measured only for special values of voltage and current in a Roentgen tube.

\section{Results and conclusions}

Using soft X-rays under conditions of Auger effect [5], we obtained periodic transitions of $\mathrm{Si}$ atoms into lower metastable states with smaller distortions of the lattice and the reverse transitions. These transitions occur due to the influence of lattice vibrations on neighboring vacancies. In recent papers $[2,3]$ it was shown that after removing $\mathrm{Si}$ atoms from the sites in the Si lattice into metastable or more stable interstitial states, negatively charged vacancies are formed, which can move long distances relatively fast. These vacancies can migrate if at least two more bonds are broken [15], for which an energy of $0.35 \mathrm{eV}$ is needed at room temperature. The energy emission after transition of a Si atom from metastable state into the vacancy is about [5] $1 \mathrm{eV}$ and is more than sufficient for the motion of vacancies. Some atoms however in the metastables states, which are neighboring to excited vacancies, can transit into more stable interstitial states changing these vacancies into unexcited. The prevailing direction of these processes depends on interaction with the lattice vibrations and electrons transitions. The periodic resonant distortions of the Si lattice presented in Fig. 2 and Fig. 4 were obtained in the process of irradiation of the crystal with soft X-rays for copper anode with voltages $U=10.4 \mathrm{kV}$ and $13 \mathrm{kV}$. This periodic process of population of metastable states and generation of excited vacancies can be explained in terms of periodic excitations of a two-level system [10] formed by atoms in metastable states of Si lattice, produced by Auger effect, and lattice vibrations interference. From electron binding energy [16] $E=1844 \mathrm{eV}$ in $K$ shell of Si atom [13] and maximum intensity of continuous spectrum of X-rays [5] for wavelength $\lambda=18.517 / U$, we can evaluate the minimum anode voltage $U_{\min }=2.754 \mathrm{kV}$ required for the occurrence of Auger effect.

However, in order to obtain the periodic resonant distortions of Si lattice, a certain amount of energy should be also transmitted into the lattice for generation of periodic resonant atom transitions from metastable states into vacancies and activation of vacancy motion. For realization of this aim, appropriate currents in the Roentgen tube must be used.

The results presented in Fig. 2 and Fig. 4 indicate that square root of the mean square displacement $i$ is significantly oscillating: $i(300 \mathrm{~min})=0.08 \times$ $10^{-10} \mathrm{~m}, i(320 \mathrm{~min})=0.15 \times 10^{-10} \mathrm{~m}, i(350 \mathrm{~min})=i(300 \mathrm{~min})$ and $i(270 \mathrm{~min})=$ 
$0.05 \times 10^{-10} \mathrm{~m}, i(300 \mathrm{~min})=0.13 \times 10^{-10} \mathrm{~m}, i(315 \mathrm{~min})=i(270 \mathrm{~min})$. Using formula (2.8), we can calculate the oscillations of concentration of $\mathrm{Si}$ atoms in metastable states for both presented cases: $N_{\mathrm{mi}}(300 \mathrm{~min})=1.598 \times$ $10^{23} \mathrm{~m}^{-3}, N_{\mathrm{mi}}(320 \mathrm{~min})=1.053 \times 10^{24} \mathrm{~m}^{-3}, N_{\mathrm{mi}}(350 \mathrm{~min})=N_{\mathrm{mi}}(300 \mathrm{~min})$ and $N_{\mathrm{mi}}(270 \mathrm{~min})=3.901 \times 10^{22} \mathrm{~m}^{-3}, N_{\mathrm{mi}}(300 \mathrm{~min})=6.857 \times 10^{23} \mathrm{~m}^{-3}$, $N_{\text {mi }}(315 \mathrm{~min})=N_{\text {mi }}(270 \mathrm{~min})$. Transitions of $\mathrm{Si}$ atoms from lattice sites into metastable states and the reverse transitions involve absorption or emission of the energy amount [5] $\Delta E_{a}=1 \mathrm{eV}$ per one $\mathrm{Si}$ atom. Thus, for transitions from metastable states into vacancies or the reverse transitions we can calculate the lost or absorbed energy $\Delta E_{\mathrm{mi}}\left(t_{2}, t_{1}\right)=\left[N_{\mathrm{mi}}\left(t_{2}\right)-N_{\mathrm{mi}}\left(t_{1}\right)\right] \Delta E_{a}$.

Now we can evaluate the emitted energies for oscillations presented in Fig. 2 and Fig. 4, respectively: $\Delta E_{\mathrm{mi}}(320 \mathrm{~min}, 300 \mathrm{~min})=1.431 \times 10^{5} \mathrm{~J} / \mathrm{m}^{3}$ and $\Delta E_{\mathrm{mi}}(300 \mathrm{~min}, 270 \mathrm{~min})=1.036 \times 10^{5} \mathrm{~J} / \mathrm{m}^{3}$. We measured the saturation of Si sample $0.3 \times 0.3 \times 1.7 \mathrm{~cm}^{3}$ through the side $0.3 \times 1.7 \mathrm{~cm}^{2}$ with irradiated vacancies. This corresponds to the increase in the mean square displacements of atoms in Fig. 2 at $t=100 \mathrm{~min}$. Irradiation was done through the open aperture with area $0.05 \times 1.5 \mathrm{~cm}^{2}$; the irradiation dose was $D=1.9668 \times 10^{5} \mathrm{mSv} /\left(\mathrm{s} \cdot \mathrm{mA} \cdot \mathrm{m}^{2}\right)$, measured with LiF thermoluminescent dosimeter [17]. After irradiation time $t=100 \mathrm{~min}$, it was found that the square root of the mean square displacement was $i=0.13 \times 10^{-10} \mathrm{~m}$, and from $(2.8)$ we obtain $N_{\mathrm{mi}}(100 \mathrm{~min})=6.065 \times 10^{23} \mathrm{~m}^{-3}$. Taking into account the volume of a sample and the irradiation dose $D$, we calculated the energy absorbed by the sample: $\Delta E=9.4612 \times 10^{-2} \mathrm{~J}$. The number of produced vacancies is $9.2795 \times 10^{16}$, and energy used for producing one vacancy with soft X-rays in the sample is $6.3644 \mathrm{eV}$. The realistic vacancy producing energy [5] is about $1 \mathrm{eV}$ and in our case we see that most of energy absorption is related to lattice excitations of Si crystal. We think that relaxation processes in $\mathrm{Si}$ crystals excited by soft X-rays is one of main reasons of very fast diffusion, or superdiffusivity [10], of vacancies, which has been measured in [18], as well as the electron-hole recombination in Si crystals [19]. However, in the latter work, like in later papers [20-22], vacancies were obtained by irradiation with 1-3 MeV electrons, gamma rays or fast neutrons. In our case, we obtained superdiffusion [10] of metastable excited vacancies generated with soft X-rays $[2,3]$ as a consequence of Auger effect [23]. The diffusion coefficients for these excited vacancies [18, 23] are $10^{4}$ times greater than for the vacancies obtained by the thermal excitation. The energies of Auger electrons for $\mathrm{Si}$ are less than $1.8 \mathrm{keV}$, and those electrons can lose energy by ionization, excitation, and multiphonon nonradiative transitions [24] of atoms which are surrounding the produced vacancies. In this case we can obtain very fast excited vacancies with long lifetimes [3]. In the paper [20] it was established that a vacancy has five different charge states $V^{++}, V^{+}, V^{0}, V^{-}, V^{--}$ and increasing energies in the silicon band gap. The different ways of broken bond reconstruction are the reason of lattice relaxation [20] which was studied in this paper. Levels for $V^{++}, V^{+}$are $0.03 \mathrm{eV}$ and $0.13 \mathrm{eV}$ above the valence band 
edge. Taking care that the minimum indirect energy gap at $300 \mathrm{~K}$ for Si crystal is $1.12 \mathrm{eV}$ we can evaluate that the energies of electrons transitions from conductivity band to these vacancies are sufficient for generation of very fast negatively charged vacancies and large lattice relaxation which we found experimentally. Also these periodic processes depend on different velocities of diffusion of generated vacancies in different charge states, dependence of Fermi level and density of charge carriers on time. According to Van Vechten [15] the initial part of dependence of values of vacancy migration from temperature can be explained introducing short-ranged forces breaking the covalent bonds and long range forces. We can suppose that in our case we have the similar situation. The very fast oscillations of intensity of reflected photons presented in Fig. 1 and Fig. 3 depend on short-ranged forces and slow oscillations of middle mean square displacements presented in Fig. 2 and Fig. 4 depend on long-ranged forces acting in lattice.

The main conclusion following from the results presented in $[10,18]$ is the relation between superdiffusivity of vacancies and lattice relaxation of Si crystals excited by soft X-rays.

\section{References}

[1] B.N. Mukashev, X.A. Abdulin, Y.V. Gorelkinski, Adv. Phys. Sci. 170, 143 (2000) (in Russian).

[2] R. Purlys, A.J. Janavičius, A. Mekys, S. Balakauskas, J. Storasta, Lith. J. Phys. 41, 376 (2001).

[3] A.J. Janavičius, J. Banys, R. Purlys, S. Balakauskas, Lith. J. Phys. 42, 337 (2002).

[4] A.I. Slucker, Y.I. Polikarpov, K.V. Vasiljeva, Phys. Solid State 44, 1529 (2002).

[5] A.F. Chochlov, Physics of Slid State, Vol. 1, High School, Moscow 2001, p. 365, (in Russian).

[6] V.S. Vavilov, A.E. Kiv, O.R. Nijazova, Mechanism of Producing and Migration of Defects in Semiconductors, Science, Moscow 1981, p. 368.

[7] G.D. Watkins, Mater. Sci. Semicond. Proc. 3, 227 (2000).

[8] L.F. Makarenko, Phys. Rev. B, Condens. Matter 308, 465 (2001).

[9] T.R. Mattson, A.E. Mattson, Phys. Rev. B 66, 214110 (2002).

[10] A.J. Janavičius, Acta Phys. Pol. A 93, 505 (1998).

[11] W. Bardyszewski, Yevick, J. Appl. Phys. 58, 2713 (1985).

[12] L. Pavesi, J. Phys., Condens. Matter 15, 1169 (2003).

[13] Ant Ural, P.B. Griffin, J.D. Plummer, Appl. Phys. Lett. 79, 4328 (2001).

[14] R. Givens, O.F. Alcantara Bonfim, Am. J. Phys. 71, 87 (2003).

[15] J.A. Van Vechten, Phys. Rev. B 10, 1482 (1974).

[16] R. Karazija, The Theory of X-ray and Electronic Spectra of Free Atoms. An Introduction, Mokslas, Vilnius 1987, p. 274.

[17] R. Purlys, Ž. Norgèla, B. Gricienè, J. Zaveckienè, Sci. helth 2, 64 (2002) (in Lithuanian). 
[18] A.J. Janavičius, Ž. Norgèla, R. Purlys, Acta Phys. Pol. A 104, 459 (2003).

[19] J.A. Van Vechten, Phys. Rev. B 38, 9913 (1988).

[20] G.D. Watkins, Mater. Sci. Semicond. Proc. 3, 227 (2000).

[21] B. Campbell, W. Choudhury, A. Mainwood, M. Newton, G. Davies, Nucl. Instrum. Methods A 476, 680 (2002).

[22] C.A. Londos, G.I. Georgiou, L.G. Fytros, N. Sarlis, Diffus. Defect Data, Solid State Data B, Solid State Phenom. 47, 281 (2004).

[23] A.J. Janavičius, Ž. Norgèla, R. Purlys, Eur. Phys. J. Appl. Phys. 29, 127 (2005).

[24] P. Landsberg, Recombination in Semiconductors, Cambridge University Press, Cambridge 2003, p. 595. 